\title{
China-UK-Tanzania Pilot Project on Malaria Control
}

\author{
Xuejiao $\mathrm{Ma}^{1}$; Shenning Lu' ${ }^{1}$; Duoquan Wang, ${ }^{1, * ;}$ Zhengbin Zhou'; Jun Feng'; He Yan'; \\ Shang Xia'; Wei Ding'; Ning Xiao'; Xiao-nong Zhou ${ }^{1}$
}

\section{KEY APPROACHES OF THE PILOT PROJECT}

The China-UK-Tanzania Pilot Project on Malaria Control supported by the China-UK Global Health Support Programme and funded by the UK Department for International Development (DFID) is the first pilot project of its kind for the Chinese government in Africa. The encouraging results of this pilot project demonstrated that the malaria burden could be reduced by $81 \%$ (1) when China's experience with malaria control was shared in Tanzania through interactions between health officials from China and Tanzania. The pilot strategy concept was implemented after China's process for malaria control and elimination was shared with Tanzania's health experts. This study's purpose was to document the essential approaches of the pilot strategy including localoriented interventions, onsite technical support from Chinese staff who worked with local health staff, and grassroots capacity-building through the sharing of China's village-doctor model anchored in African local contexts.

In the pilot project, China's experience with malaria control was shared via local-oriented and evidencebased interventions (2). The baseline survey conducted in Rufiji District of Tanzania in 2015 was implemented by local health staff with the guidance of onsite Chinese staff, who also helped to identify the malaria prevalence in the community and weaknesses in the local health system. In the baseline survey, 13 health facilities (HFs) were surveyed, of which 10 were dispensaries and 3 were health centers. Only $15.38 \%$ of the HFs were found to have stocked Dihydroartemisinin/Piperaquine. The incompleteness of HFs' routine data was also a challenge to local malaria control. Moreover, the 4,685 participants in the intervention communities had a malaria prevalence of $25.7 \%$ based on a rapid diagnostic test (RDT), in contrast with the locally reported malaria prevalence rate of $16.0 \%$ (3). This data discrepancy provided crucial evidence that additional information was necessary regarding the local surveillance system.

Following the baseline survey, Chumbi and Ikwiriri were selected as intervention communities while Bungu and Kibiti served as control communities (4). The locally-tailored 1,7-Reactive Community-Based Testing and Response (1,7-RCTR) was the main intervention in the selected communities that was administered in addition to existing malaria control and prevention implemented by the Ministry of Health through the National Malaria Control Program (NMCP). Two control communities were monitored that received no interventions beyond those provided by the NMCP. The pilot project was designed to improve rather than to alter the existing processes in the local health systems of the intervention communities. Specifically, the 1,7-RCTR implemented at the village level entailed reporting all confirmed malaria cases at the HFs within 24 hours followed by screening and treatment of identified hotspots within the next week to reduce malaria burden. This targeted intervention aligns with the World Health Organization (WHO)'s high-impact initiative for countries with moderate and high transmission by tailoring the Chinese experiences and WHO-T3 initiative (Test, Treat, Track.) to the local settings of Tanzania (5). In the intervention communities, locally tailored interventions based on the 1,7-RCTR were designed and implemented, including onsite Chinese technical support, capacity building for local community health workers (CHWs), and multilateral collaboration.

Unlike some international projects, the onsite Chinese staff for technical support were dispatched to work with local stakeholders for the duration of the pilot project in a mutual learning-by-doing approach. Chinese staff trained in malaria epidemiology, laboratory systems, vector control, and information systems were selected to collaborate with the Ifakara Health Institute (IHI) and other local partners (G). Each Chinese staff member was paired with a local staff member to work on a discreet aspect of the intervention and make implementation decisions in 
the field, mainly by supervising and guiding local training and field operations. The teams conducted community mobilization campaigns with over 500 local stakeholders including local government leaders and health staff from 36 administrative villages in the 4 communities (G). Additionally, the teams ensured the steady progress of the project's implementation in the field. Capacity-building through the sharing of China's village-doctor model cultivated a local team of 35 CHWs, who were trained and supervised and ensured the success of the implementation process. The training included case management, vector control, and health education. China's village-doctor model was shared in such a way that CHWs could provide basic malaria diagnosis, drug treatment, and primary health education to members of the community, even reaching previously under-served patients. Multilateral collaboration and resource integration were key drivers in maximizing all parties' efforts. In the pilot project, resources from China were integrated with Tanzania NMCP for efficient utilization. For example, although long lasting insecticidal nets (LLINs) delivery was not supported by the project funding, around 50,000 LLINs were allocated by NMCP to reduce the risk of local malaria transmission (7).

To evaluate the pilot strategy in Tanzania, the endline survey was conducted in 2018 with 4,406 people in the intervention communities and 5,728 in the control communities. The malaria infection rate in the intervention communities was $4.9 \%$, representing a decline of $81 \%$, while the malaria infection rate decreased by $52 \%$ in the control communities. The dramatic reduction in the intervention wards compared to the control areas produced clear evidence that the malaria prevalence was reduced beyond the impact of NMCP alone. Currently, other malaria interventions, including the most advanced novel vaccination approaches, have only reported limited effects beyond that of LLIN use (8).

\section{EXPERIENCES SHARED IN THE PILOT PROJECT}

The pilot project entailed the sharing of China's experience with malaria control in African settings through the pilot strategy. The pilot strategy has been continuously used in China's malaria control and elimination process and has proved conducive to the selection of local effective measures, cultivation of the local team's capacity, and the enrichment of work experience regarding malaria control. Therefore,
China's experience on malaria control could be shared in additional settings.

Learning by doing: Learning by doing was explored in the pilot project as a problem-solving process. Chinese staff worked together with IHI field teams in the pilot communities to identify the obstacles in the malaria control process and resolve them. Onsite Chinese staff were paired with local staff from IHI, NMCP, and other partners and jointly worked on a specific aspect of the project for work plan designing, local staff training, field implementation, and supervision. The use of a collaborative model and teamwork realized through regular lectures on malaria control and internal assessments as well as quarterly stakeholders' meetings led to a mutual understanding of the goals of the pilot project. Thanks to the collaborative efforts achieved through community consent, community mobilization, and community participation, as well as the local leaders' and IHI's support, the pilot project was successfully implemented.

Sharing China's village-doctor model: Capacity building for CHWs through the community-based approach to malaria control was constructive in mobilizing community participation and project implementation. The name "barefoot doctor" was replaced by "village doctor" in China in 1981 (9). Village doctors have made great contributions to malaria control by providing primary health care to malaria patients in rural areas at relatively low costs in a door-to-door manner (10). Similarly, CHWs play important roles in delivering quality primary health care services and malaria control efforts, which are recognized by the World Health Assembly and promoted according to the WHO strategy (11). Many people do not go to health facilities due to a lack of awareness of malaria illness, accessibility of health services, and concern regarding high treatment costs. This project explored the feasibility of a communitybased approach (4) through the sharing of China's village-doctor model in Tanzania. CHWs were recruited from local communities and their door-todoor healthcare services helped ensure the primary health of community members and served as an extension of the services provided by local health facilities.

Multilateral cooperation: Multilateral cooperation was indispensable for the effective implementation of the pilot project, which involved 11 main stakeholders from China, Tanzania, and international organizations. The internal evaluation was led by Duke University, 
midterm and final evaluations were led by the WHO, and the external evaluation was led by the DFID. In this learning-by-doing process, the different stakeholders worked together to identify adapted approaches for sharing China's experience with malaria control and elimination (12).

The pilot project also encountered some challenges that could provide insight for future public health cooperation between China and Africa. China has no template to guide its engagement in global health initiatives (13). Therefore, two approaches should be considered: 1) fostering systematic changes in its international health cooperation system; and 2) working with other global agencies to build new global health partnerships. Future public health cooperation should be maintained and nurtured between China and Africa to benefit Tanzania and other places.

Acknowledgments: The authors are grateful to all partners from Chinese, Tanzanian, and international organizations who made a contribution to the pilot project. We also appreciate Prof. Salim Abdulla, Dr. Lewis Husain, Dr. Qin Chen, and Dr. Prosper Chaki for their help in improving the manuscript.

doi: $10.46234 / \mathrm{ccdcw} 2020.179$

\# Corresponding author: Duoquan Wang, wangdq@nipd.chinacdc.cn.

\footnotetext{
${ }^{1}$ National Institute of Parasitic Diseases, Chinese Center for Disease Control and Prevention; Chinese Center for Tropical Diseases Research; WHO Collaborating Centre for Tropical Diseases; National Center for International Research on Tropical Diseases, Ministry of Science and Technology; Key Laboratory of Parasite and Vector Biology, Ministry of Health, Shanghai, China.
}

Submitted: July 03, 2020; Accepted: August 10, 2020

\section{REFERENCES}

1. WHO. Fostering the China-Africa cooperation for the elimination of Malaria. https://www.afro.who.int/news/fostering-china-africa-coopera tion-elimination-malaria. [2020-8-1].

2. China Malaria Control and Research Committee. Malaria control and research in China. Beijing: People’s Medical Publishing House. 1991.

3. National Malaria Control Programme. Malaria surveillance bulletin 2017. Tanzania: Dar es Salaam. 2017. https:/www.measureevaluation. org/measure-evaluation-tz/malaria/Malaria\%20Bulletin\%20issue\%204. pdf. [2020-8-1].

4. Wang DQ, Chaki P, Mlacha Y, Gavana T, Michael MG, Khatibu R, et al. Application of community-based and integrated strategy to reduce malaria disease burden in southern Tanzania: the study protocol of China-UK-Tanzania pilot project on malaria control. Infect Dis Poverty 2019;8:4. http://dx.doi.org/10.1186/s40249-018-0507-3.

5. World Health Organization, RBM Partnership to End Malaria. High burden to high impact: a targeted malaria response. 2018. https://apps. who.int/iris/bitstream/handle/10665/275868/WHO-CDS-GMP-2018. 25-eng.pdf?ua=1. [2020-08-01].

6. China Youth Daily. Malaria control in Africa. 2018. https://k.sina.com. cn/article_1726918143_66eeadff02000f6ek.html. [2020-08-01]. (In Chinese).

7. China CDC News. A new chapter in malaria prevention and control in aid to Africa: the cooperation between China, Britain and Tanzania in malaria prevention and control has achieved initial results! 2017. https://dy.163.com/article/CP8G6QVT05149B3T.html;NTESwebSI= 2CD5E76D1AC965EFFF712C299E9AE9E9.hz-subscribe-webdocker-cm-online-rpqqn-8gfzd-di161-6785987mg4p-8081. [2020-0801]. (In Chinese).

8. Maher B. Malaria: the end of the beginning. Nature 2008;451:1042 6. http://dx.doi.org/10.1038/4511042a.

9. de Geyndt W, Zhao XY, Liu SL. From barefoot doctor to village doctor in rural China (English). World Bank Technical Paper; No. WTP 187. Washington, DC: World Bank Group; 1992. http://documents. worldbank.org/curated/en/783641468743703155/From-barefootdoctor-to-village-doctor-in-rural-China. [2020-08-01].

10. Zhang DQ, Unschuld PU. China's barefoot doctor: past, present, and future. Lancet 2008;372(9653):1865 - 7. http://dx.doi.org/10.1016/ S0140-6736(08)61355-0.

11. World Health Organization. Momentum for community health workers at the Seventy-second World Health Assembly. Geneva: World Health Organization. https://www.who.int/news-room/detail/22-052019-momentum-for-community-health-workers-at-the-seventysecond-world-health-assembly. [2020-8-1].

12. Ma XJ, Ding W, Wang DQ, Duan L, Huang LL, Wang B, et al. Main achievements and challenges of China-UK-Tanzania Pilot Project on Malaria Control. Chin J Parasitol Parasit Dis 2020;38(3): 360. http://kns.cnki.net/kcms/detail/31.1248.R.20200608.1441.006. html. (In Chinese).

13. Husain L, Bloom G, McPherson S. The China-UK Global Health Support Programme: looking for new roles and partnerships in changing times. Glob Health Res Policy 2020;5:26. http://dx.doi.org/ 10.1186/s41256-020-00156-1. 